\title{
A Metal Matrix CNTS Modified Electrode Fabricated Using Micromachining-Based Implantation Method for Improving Sensitivity and Stability
}

\author{
Yan Wang, Chen Ran, Yuan Yang, Guifu Ding, and Hong Wang \\ National Key Laboratory of Science and Technology on Micro/Nano Fabrication Shanghai Jiao Tong University, \\ Shanghai 200240, China
}

Correspondence should be addressed to Yan Wang; wyyw@sjtu.edu.cn

Received 21 August 2013; Revised 29 September 2013; Accepted 22 October 2013

Academic Editor: Shiren Wang

Copyright (C) 2013 Yan Wang et al. This is an open access article distributed under the Creative Commons Attribution License, which permits unrestricted use, distribution, and reproduction in any medium, provided the original work is properly cited.

\begin{abstract}
The metal matrix carbon nanotubes modified electrode (MCME) has been fabricated by a novel process involving preparation of carbon nanotubes (CNTs)/polyimide (PI) composite film, wet, etching, sputtering, electroplating, and wet-etch releasing. Pretreated CNTs are dispersed in PI by mechanical ball milling and then CNTs solution is spin-coated on the substrate. The CNTs/PI composite film is etched away a layer of PI to expose tips of CNTs using buffering solution. These exposed tips of CNTs are covered by metal particles in sputtering process as metal seed layer, followed by metal supporting film formed by electroplating. The MCME is obtained after releasing PI film from the metal supporting film. The MCME shows well morphology of uniform distributional protruding tips of CNTs and increased electron transfer efficiency with strong bonding connection between CNTs and metal matrix, which greatly improves sensitivity and stability of the MCME. The oxidation peak of the MCME in cyclic voltammeter (CV) test is 1.7 times more than that of CNTs suspension spin-coated metal electrode (SCME). The decline of peak current of the MCME after fifty cycles is only $1.8 \%$ much less than $67 \%$ of the SCME. Better sensitivity and stability may be helpful for CNTs modified electrodes wide application for trace test of many special materials.
\end{abstract}

\section{Introduction}

The CNTs are widely used in electrochemical analysis $[1,2]$ because of its combination of high aspect ratio, nanometersized dimensions, good electrical conductivity [3], and low capacitance in the pristine state. CNTs modified electrodes were first introduced for voltammetric analysis by Liu et al. [4]. Following this report, the electrocatalytic properties of CNTs modified electrodes became a hot topic. The specific area and the number of catalytic sites of electrode can be increased by compositing CNTs on the electrode. The basic electrode configuration of randomly dispersed, purified CNTs on the surface of a conducting support macro electrode was used extensively for the electrochemical detection of a multitude of redox-active molecules in solution [5]. However, the most studies put key point on sensitivity of CNTs to different materials. Limited effort about fabrication method was made to increase the sensitivity and stability of the modified electrode.
Preparation methods of CNTs modified electrode can be concluded two kinds: one is CNT suspension and the other one is CNT/polymer composite. Each method has certain advantage and disadvantages. For example, CNTs in suspension have good dispersibility, and easily be spin-coated on the substrate after solution volatilizing, clear reflecting surface can be obtained. Rezaei and Mirahmadi Zare dropped the suspension of multiwall carbon nanotubes (MWNTs) in nitric acid on the cleaned glassy carbon electrode (GCE) and then removed the acid to gain a modified glassy carbon with a high sensitivity to noscapine [6]. The similar method was used to prepare functionalized multiwall carbon nanotubes modified glassy carbon electrodes for the simultaneous determination of dopamine and acetaminophen [7]. However, CNTs are distributed on the metal substrate with morphologies of cluster and single one. In addition, CNTs connect with metal matrix by van der Waals force. Weak connection results in the big electric resistance and makes CNTs easily fall down from the substrate. Contaminates 
produced in test process will adhere to the surface of the CNTs. After treatments for dislodging such contaminates cannot be effectively carried out because of weak connection between CNTs and substrate. Frequently used methods, such as ultrasonic washing and chemical treatment, will lead to loss of CNTs from substrate, which impacts the lifetime and stability of CNTs modified electrode deeply. In CNT/polymer composite, polymer connects CNTs with substrate firmly by cohesive action. CNT/Nafion composite [8], poly-o-phenylenediamine (PoPD)/MWNTs composite [9], and CNTs/ethylene vinyla cetate (EVA) composite [10] were used to fabricate CNTs modified electrodes. CNT/polymer composite film is usually fabricated by dispersion, spincoating, solidification, and etching. CNTs firstly are dispersed in polymer by mechanical ball milling and then spin-coated on conductive substrate. Solidification is carried out to form film structure. The composite film is etched to expose parts of CNTs for forming electrical interface of the electrode. $\mathrm{P}$ CNTs are intertwined in composite film and connected with the substrate by polymer bonding layer. Compared with adsorption, such cohesion structure avoids loss of CNTs from substrate and provides better stability of electrodes. However, electrical connection between CNTs and substrate still depends on van der Waals force, which results in the same weak electrical transfer efficiency of the electrode.

In this study, we propose a metal matrix carbon nanotubes modified electrode (MCME) fabricated by micromachining-based implantation method. Pretreated CNTs and polyimide (PI) composites are spin-coated onto a substrate and protruding tips of CNTs are obtained by etching away a layer of PI. These exposed tips of CNTs are covered by metal particles in sputtering process as metal seed layer, followed by metal supporting film formed by electroplating. The MCME is obtained after releasing PI film from the metal supporting film. Through the process of micromachiningbased implantation method, CNTs are vertically embedded in the flat Ni metal matrix substrate. The CNT modified electrode we fabricated shows relatively good sensitivity and stability than traditional ones in cyclic voltammetric (CV) study. It is believed that this is the first report of CNT modified electrode fabricated by this special fabrication method and the investigation may be helpful for CNT modified electrode wide application for industry.

\section{Experimental}

Referring to Figure 1, the fabrication process of the MCME is described as follows.

(1) A glass substrate is ultrasonically cleaned by alkali $(30 \% \mathrm{KOH})$ and deionised water.

(2) Initially, multiwalled carbon nanotubes (MWNTs) with tube diameters ranging from 60 to $100 \mathrm{~nm}$ and length of $5 \sim 15 \mu \mathrm{m}$ are boiled in potassium hydroxide molten for $5 \mathrm{~h}$ and concentrated sulfuric acid for $21 \mathrm{~h}$ in order to obtain pure and dispersed materials. Then, the pretreated MWNTs and PI with mass ratio of 1:9 are mixed by grinding and mechanical ball milling at $50 \mathrm{~Hz}$ for $5 \mathrm{~h}$ to produce a homogeneous composite.

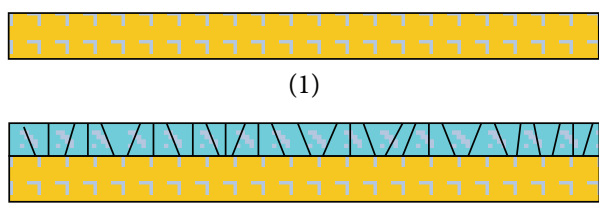

(2)

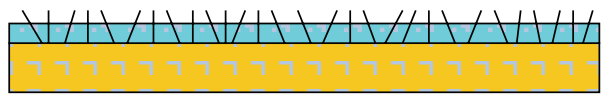

(3)

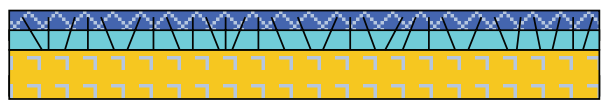

(4)

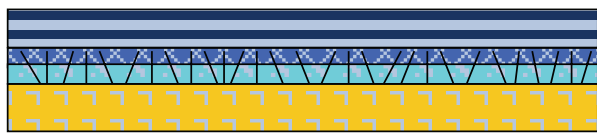

(5)

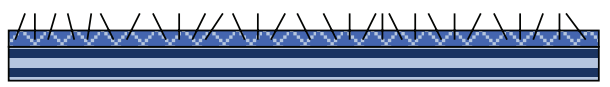

(6)

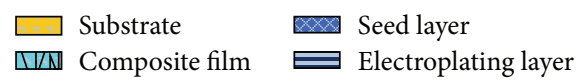

FIGURE 1: The fabrication process of MCME: (1) cleaning a glass substrate, (2) forming CNT/PI composite film, (3) wet chemical etching of PI layer, (4) sputtering $\mathrm{Cr} / \mathrm{Cu}(30 / 50 \mathrm{~nm})$ conducting layer, (5) electroplating of the Ni film, (6) releasing the PI and peel off the $\mathrm{Ni}$ film.

In this process, dimethylacetamide (DMAC) is used as solvent for diluting PI. Finally, the CNTs and PI composite is deposited on the glass substrate by spincoating with rotation speed of $1000 \mathrm{rpm}$ to form $20 \mu \mathrm{m}$ thick film and then is dried under programmed temperature mode to $110^{\circ} \mathrm{C}$.

(3) A layer of PI is etched away from the MWNTs/PI composite film to obtain protruding tips by the buffer solution $(\mathrm{ph}=11.4,10.5)$ made of phosphate. The etching depth is carefully controlled by etching time in order to keep the roots of CNTs that still remain in the composite film. $\sim 0.1 \mu \mathrm{m} / \mathrm{min}$ and $\sim 1.5 \mu \mathrm{m} / \mathrm{min}$ etching rate are obtained by ultrasonic stirring and natural convection, respectively. $\sim 5 \mu \mathrm{m}$ thick PI film is etched by appropriate combination of ultrasonic stirring and natural convection.

(4) The metal seed layer of $\mathrm{Cr} / \mathrm{Cu}(30 / 50 \mathrm{~nm})$ is deposited on the above etched surface to cover the protruding tips of CNTs by sputtering.

(5) The supporting metal film of $\mathrm{Ni}(100 \mu \mathrm{m})$ is deposited on the above metal seed layer by electroplating (the reason for choosing $\mathrm{Ni}$ is its resistance to corrosion).

(6) $\mathrm{PI}$ is released by $2 \% \mathrm{NaOH}$ in magnetic stirring reactor until the supporting metal film can be peeled off easily. Protruding tips of CNTs on the Ni film surface is observed by SEM and tested by energy dispersive spectrometer (EDS) to ensure whether PI is released 


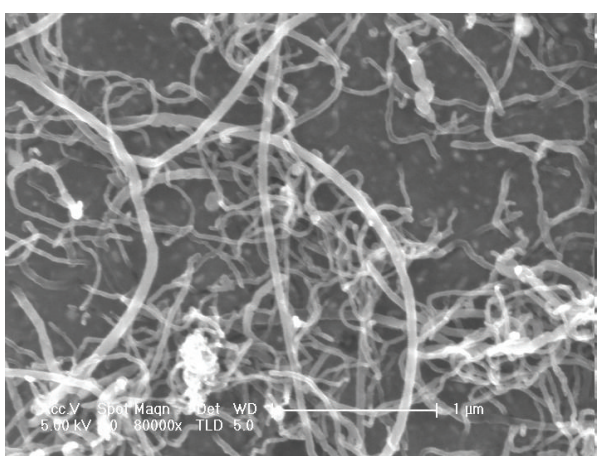

(a)

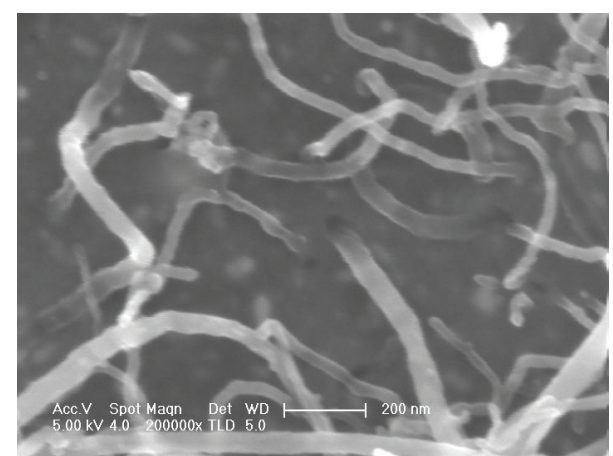

(b)

FIgURE 2: The SEM images of the MCME with different amplified times: (a) 80000X and (b) 200000X.

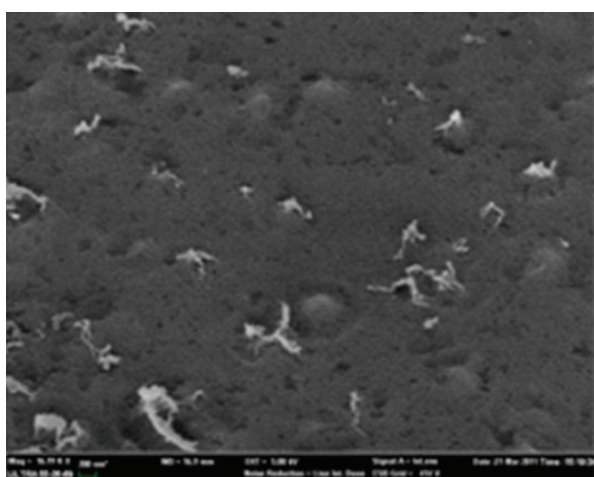

(a)

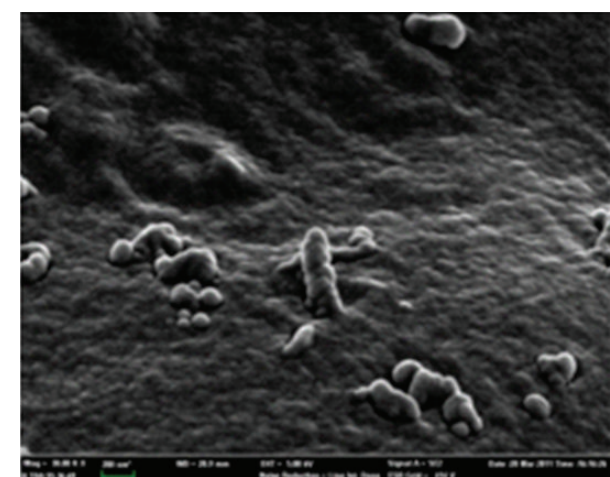

(b)

FIGURE 3: The SEM images of the MCME before (a) and after (b) sputtering process.

completely. Releasing process is repeated for clearing protruding tips of the CNTs, which helps obtain the excellent reaction interface of the electrode.

MWNTs (diameter $60 \sim 100 \mathrm{~nm}$, length $5 \sim 15 \mu \mathrm{m}$, the purity is more than 95\%) are purchased from Shenzhen Nanotech Port Co. Ltd. (Shenzhen, China). PI (Number ZKPI-510) is purchased from POME Sic-tech. Ltd., Beijing (China). All chemicals are of analytical grade and are used without further purification. All experimental steps stated above in (1)-(6) are completed at room temperature $\left(\sim 25^{\circ} \mathrm{C}\right)$. Scanning electron microscope (SEM) and energy dispersive spectrometer (EDS) (JSM-6700F of JEOL Company, Germany) are used to analyze the surface morphology and component analysis of the MCME.

\section{Results and Discussion}

Figure 2 shows a series of SEMs of the MCME with different amplified times. It can be seen that the MCMEs with a flat surface are covered with dispersed CNTs as shown in Figure 2(a). The roots of CNTs are firmly embedded in the metal layer and no contaminant is induced (see Figure 2(b)). The connection between CNTs and substrate are surface absorption in both CNT suspension and CNT/polymer composite film. Metal particles in sputtering process are injected into inner and outer surfaces of CNTs forming a covered metal seed layer in the MCME. Figure 3 shows the two SEMs before and after sputtering process. It is clear that the protruding tips of the CNTs are covered by a continuous and smooth metal seed layer after sputtering process. Metal ions in solution are deposited on the metal seed layer through electroplating process. $\sim 150 \mu \mathrm{m}$ thick supporting metal film with enough self-supporting strength is obtained. The roots of the CNTs are deeply embedded in the supporting metal matrix, which induces strong mechanical connection and low electric contact resistance. The decreased electric contact resistance reveals the apparently high peak current and low reaction voltage in electrical chemical test.

The cyclic voltammetric (CV) study is performed using CHI-660 electrochemical workstation (Chenhua Instrument Company, China) with a standard three electrode configuration (as shown in Figure 4). The CNTs suspension spincoated metal electrode (named SCME), the Ni electrode (named NE), and MCME are prepared as working electrodes. Commercial MWCTs alcohol suspension (diameter 60 $100 \mathrm{~nm}$, length $5 \sim 15 \mu \mathrm{m}$, purity $>95 \%$, ash $<2 \%$, and mass ratio 1:50) purchased from Shenzhen Nanotech Port Co. Ltd. (Shenzhen, China) is drop-coated on a bare Ni electrode. Alcohol is volatilized in heating furnace under $50^{\circ} \mathrm{C}$. Drop coating and volatilizing processes are repeated at least five times to ensure a sufficient amount of MWCTs on the surface 


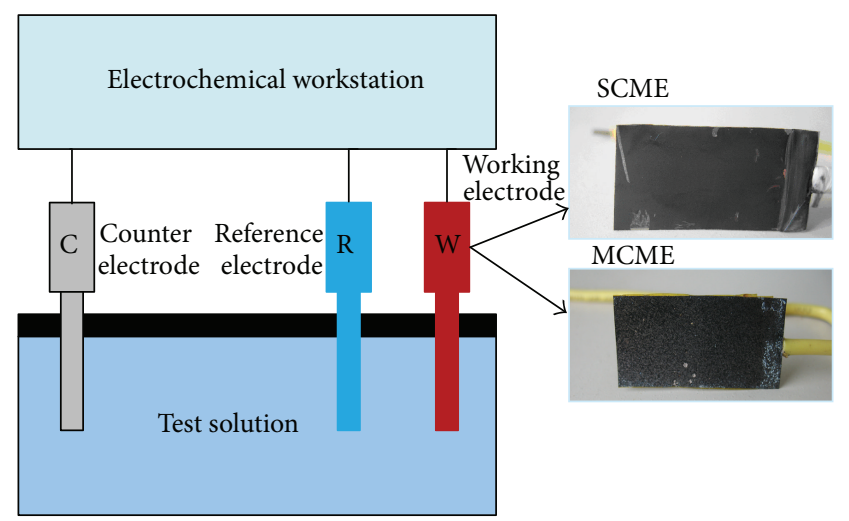

FIGURE 4: Sketch of the test setup for the SCME and the MCME.

of the SCME. The aqueous solution of $200 \mathrm{~mL}$ containing $\mathrm{Cu}_{2} \mathrm{P}_{2} \mathrm{O}_{7}(60 \mathrm{~g} / \mathrm{L})$ and $\mathrm{K}_{4} \mathrm{P}_{2} \mathrm{O}_{7}(200 \mathrm{~g} / \mathrm{L})(\mathrm{PH}$ is adjusted to 7.6 by $\mathrm{C}_{6} \mathrm{H}_{8} \mathrm{O}_{7}$ ) is added to the test electrolytic bath. The oxidation current peaks of the copper ions that occur at $\mathrm{CV}$ curves mainly depend on electron transfer at the interface between the electrode and solution. The highly increased electron transfer efficiency at the interface increases the peak current and leads to the reduction of the corresponding potential.

Figure 5 shows the curves of peak current corresponding to the oxidation of copper ions on the NE, the SCME, and the MCME. The peak of $-0.007 \mathrm{~A}$ corresponding to the oxidation of copper ions on metal matrix surface appears $\sim 0.02 \mathrm{~V}$ on the NE. CNTs induced on electrode might be attributed to the increased surface charge storage, which not only decreases the potential of peak current but also increases the peak current. The peak of $-0.042 \mathrm{~A}$ corresponding to the oxidation of copper ions on CNTs surface appears at $-0.12 \mathrm{~V}$ on the SCME. However, a reflecting potential barrier appears because of weak electric contact between CNTs and metal matrix on the SCME, which decreases electrons transfer efficiency of the oxidation of copper ions on CNTs surface. The peak current corresponding to the oxidation of copper ions on metal matrix surface $\sim 0.02 \mathrm{~V}$ (the same to the potential on the NE) still exists on the SCME with current of $-0.0125 \mathrm{~A}$. Lower electric contact resistance in the MCME increases electron transfer efficiency between CNTs and metal matrix. The peak of $-0.074 \mathrm{~A}$ appears at $-0.072 \mathrm{~V}$ in CV curve of the MCME. The potential of $-0.072 \mathrm{~V}$ at the peak current is lower than $-0.12 \mathrm{~V}$ of the SCME. In addition, the oxidation current peak of the copper ions on metal surface disappears because the oxidation reaction on CNTs' surface in the MCME is more efficient than that on the metal surface. Most copper ions react with electrons at CNTs surface with the higher electrons transfer efficiency and the lower reflection potential barrier. So there is the only one peak current peak $(-0.074 \mathrm{~A})$ appearing in the MCME with 1.7 times higher than $-0.042 \mathrm{~A}$ in the SCME.

Strong connection between CNTs and metal matrix increases the sensitivity of the MCME and improves the stability of the electrode. CV tests of fifty cycles are carried out on the SCME and the MCME as shown in Figure 6, respectively.
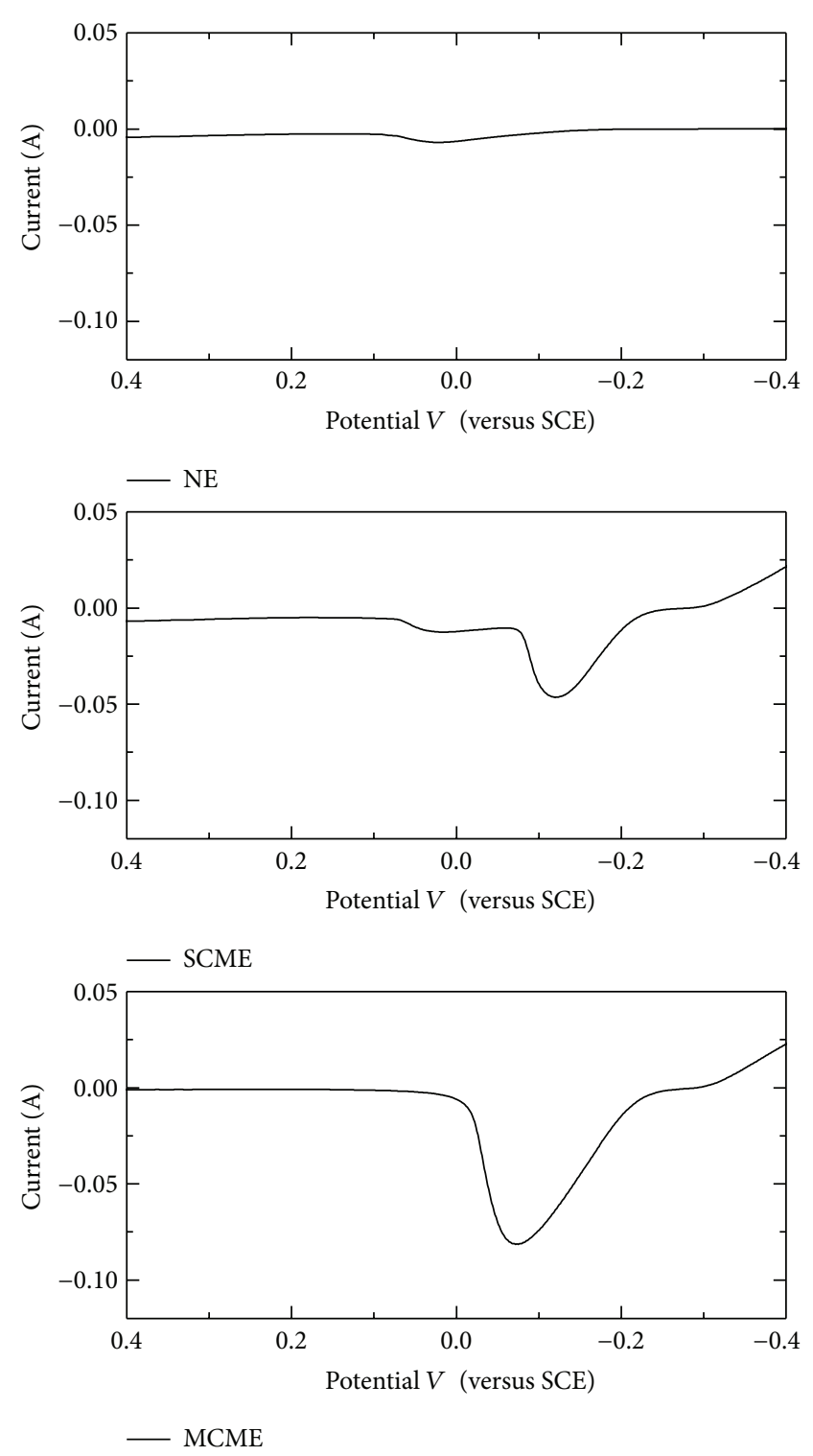

Figure 5: The CV curves of the NE, the SCME, and the MCME in $\mathrm{Cu}_{2} \mathrm{P}_{2} \mathrm{O}_{7}$ solution.

CNTs drop down from the substrate of the SCME through the test process because of their weak connection with substrate, which induces successive decreased peak current in each cycle (see in Figure 6(a)). CNTs suspension forms adsorption layers on the substrate. The adsorption layers drop down layer by layer for infiltration of CNTs with solution. The decline amplitude of the peak current is large at the beginning cycles and keeps stable when the CNTs' adsorption is equilibrium with infiltration of CNTs as shown in Figure 6(a). The peak current decline is $\sim 67 \%$ on the CV curves of the SCME after fifty cycles. CNTs are firmly embedded in metal matrix and are exposed protruding tips on the surface of the MCME. Good connection between CNTs and metal matrix results from high injection energy of metal ions and covered metal layer on CNTs' surface. The peak current changed from $-0.06952 \mathrm{~A}$ to $-0.0683 \mathrm{~A}$ after fifty cycles for only $\sim 1.8 \%$ decline (shown in Figure 6(b)). 


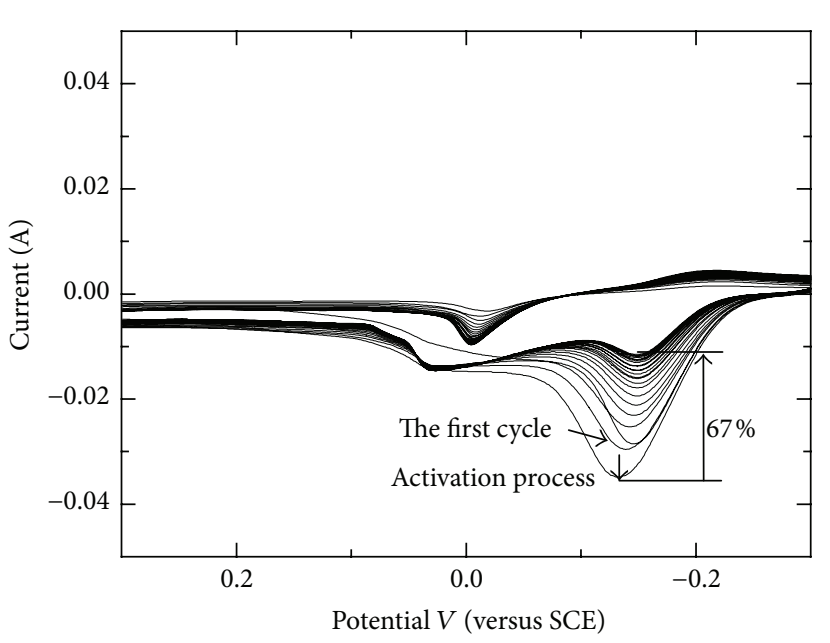

(a)

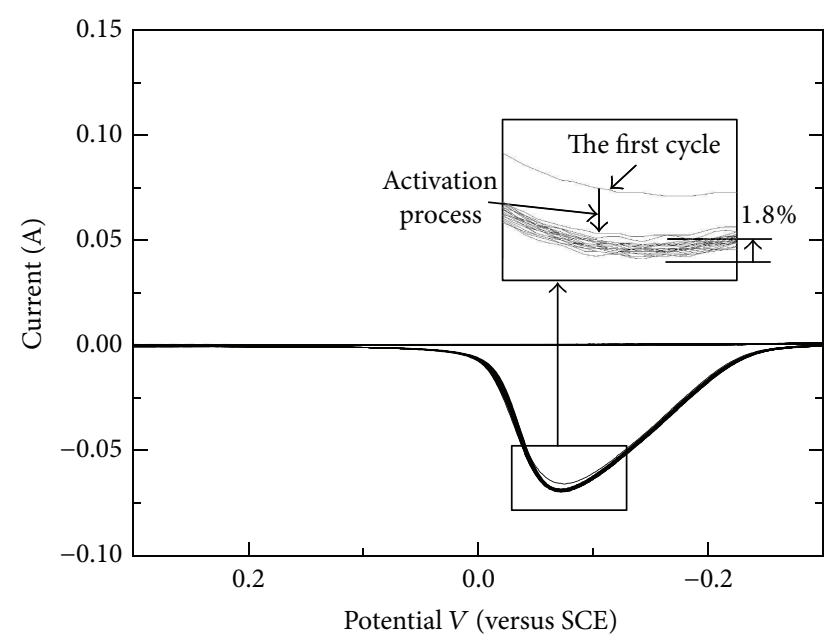

(b)

Figure 6: The fifty CV cycles of the SCME (a) and the MCME (b) in $\mathrm{Cu}_{2} \mathrm{P}_{2} \mathrm{O}_{7}$ solution.

\section{Conclusion}

The metal matrix carbon nanotubes modified electrode fabricated using micromachining-based implantation method is achieved. The relevant processing technology is also developed. The MCME has strong bonding force between carbon nanotubes and metal matrix. The structure reveals a very efficient performance as indicated by the high oxidation peak current and stability. Better sensitivity and stability may be helpful for carbon nanotubes modified electrode wide application for trace test of many special materials. This study laid a foundation for the implanted carbon nanotubes modified electrode mass fabrication, but it requires further optimization in carbon nanotubes morphology control and effective post treatment.

\section{Acknowledgments}

The authors express their sincere gratitude to the colleagues of-National Key Laboratory of Nano /Micro-Fabrication Technology and thanks for their support and encouragement. The authors also express their sincere gratitude to the Chinese National Natural Science Foundation (91023029 and 51305265) and the Research Fund for the Doctoral Program of Higher Education of China (20120073110061).

\section{References}

[1] F. Valentini, A. Amine, S. Orlanducci, M. L. Terranova, and G. Palleschi, "Carbon nanotube purification: preparation and characterization of carbon nanotube paste electrodes," Analytical Chemistry, vol. 75, no. 20, pp. 5413-5421, 2003.

[2] X. Yu, D. Chattopadhyay, I. Galeska, F. Papadimitrakopoulos, and J. F. Rusling, "Peroxidase activity of enzymes bound to the ends of single-wall carbon nanotube forest electrodes," Electrochemistry Communications, vol. 5, no. 5, pp. 408-411, 2003.

[3] R. Saito, M. Fujita, G. Dresselhaus, and M. S. Dresselhaus, "Electronic structure of chiral graphene tubules," Applied Physics Letters, vol. 60, no. 18, pp. 2204-2206, 1992.
[4] C. Y. Liu, A. J. Bard, F. Wudl, I. Weitz, and J. R. Heath, "Electrochemical characterization of films of single-walled carbon nanotubes and their possible application in supercapacitors," Electrochemical and Solid-State Letters, vol. 2, no. 11, pp. 577578, 1999.

[5] I. Dumitrescu, P. R. Unwin, and J. V. MacPherson, "Electrochemistry at carbon nanotubes: perspective and issues," Chemical Communications, no. 45, pp. 6886-6901, 2009.

[6] B. Rezaei and S. Z. Mirahmadi Zare, "Modified glassy carbon electrode with multiwall carbon nanotubes as a voltammetric sensor for determination of noscapine in biological and pharmaceutical samples," Sensors and Actuators B, vol. 134, no. 1, pp. 292-299, 2008.

[7] Z. A. Alothman, N. Bukhari, S. M. Wabaidur, and S. Haider, "Simultaneous electrochemical determination of dopamine and acetaminophen using multiwall carbon nanotubes modified glassy carbon electrode," Sensors and Actuators B, vol. 146, no. 1, pp. 314-320, 2010.

[8] J. Wang, M. Musameh, and Y. Lin, "Solubilization of carbon nanotubes by Nafion toward the preparation of amperometric biosensors," Journal of the American Chemical Society, vol. 125, no. 9, pp. 2408-2409, 2003.

[9] X. Zhang, S. Wang, L. Jia, Z. Xu, and Y. Zeng, "An electrochemical sensor for determination of calcium dobesilate based on PoPD/MWNTs composite film modified glassy carbon electrode," Journal of biochemical and biophysical methods, vol. 70, no. 6, pp. 1203-1209, 2008.

[10] J. N. Wohlstadter, J. L. Wilbur, G. B. Sigal et al., "Carbon nanotube-based biosensor," Advanced Materials, vol. 15, no. 14, pp. 1184-1187, 2003. 

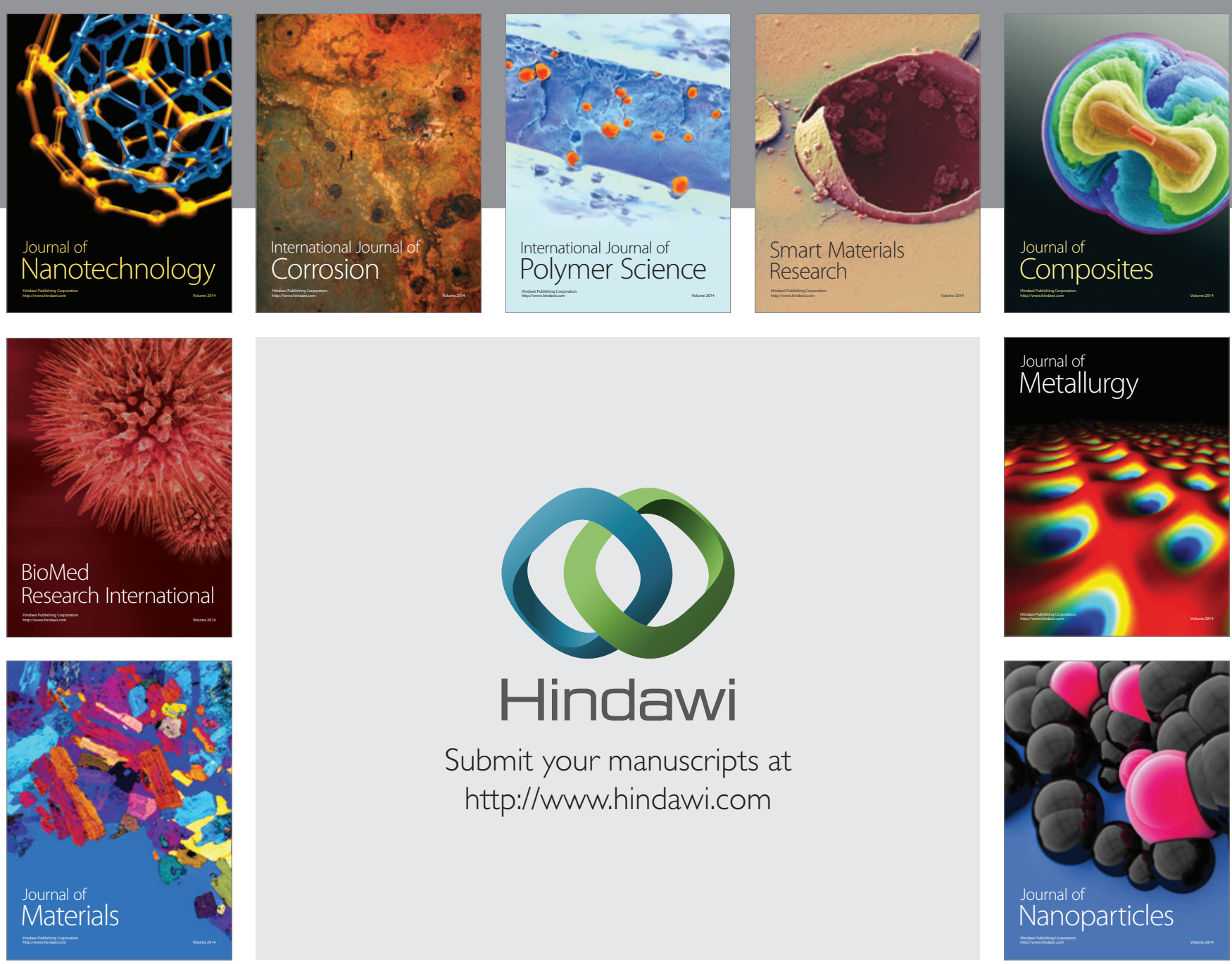

Submit your manuscripts at http://www.hindawi.com
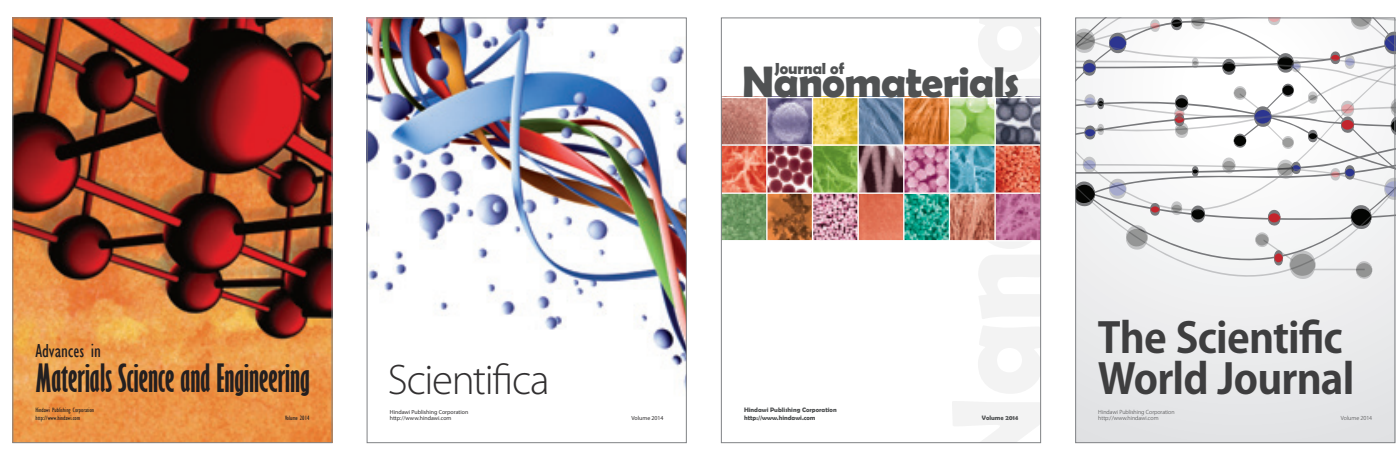

\section{The Scientific World Journal}
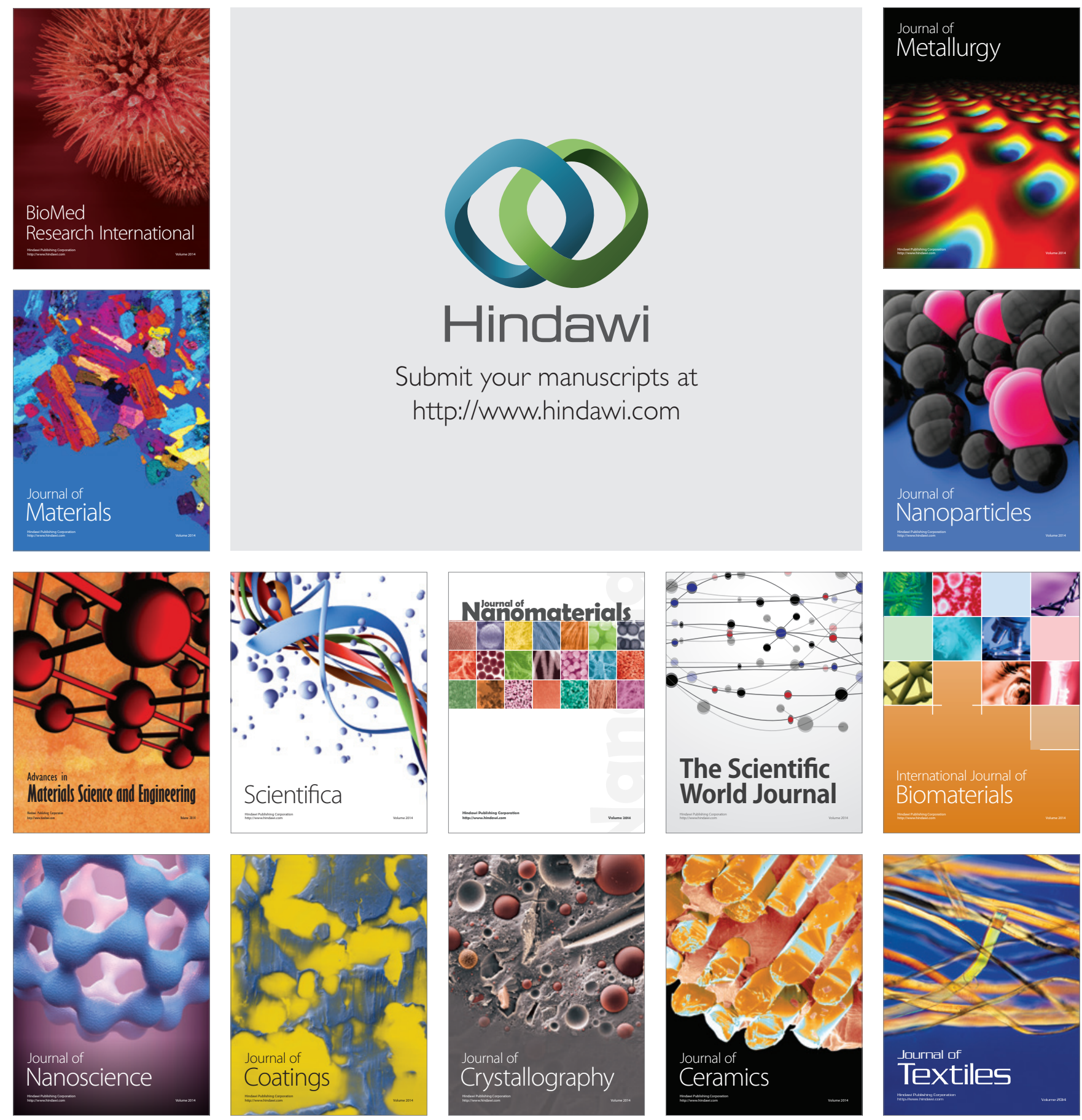